\title{
An Investigation on Zero Cutoff Frequency in a Rectangular Waveguide Periodically Loaded with MNG Metamaterial
}

\author{
E. Mobini and F. Mohaje
}

\begin{abstract}
In this paper the propagation of electromagnetic wa-ve inside a waveguide which is periodically loaded with anisotropic metamaterial with transversal negative permeability and positive permittivity (MNG) is analyzed. Using Maxwell's equations and Fluquet's theorem, dispersion equation of the structure is derived and discussed. The obtained equation is general and valid for propagating and attenuating modes in both anisotropic MNG and air medias. Our study is mainly concentrated on the existence of the zero cutoff frequency in a rectangular waveguide which is periodically loaded with MNG metamaterial .All simulations are done for two different thicknesses of MNG layers and the achieved results are compared with the results of a hollow waveguide. Results indicate that the electromagnetic dispersion curve, zero cutoff frequency, stopband and passband intervals can be highly affected by the permeability and the thickness of the layers.
\end{abstract}

Index Terms-MNG metamaterial, zero cutoff frequency

\section{INTRODUCTION}

In recent years analysis of electromagnetic wave propagation in a rectangular waveguide filled with metamaterial has been widely investigated. These materials represent some unusual properties like: anticutoff behavior, backward wave propagation[1-7] and near zero or zero cutoff frequency[8] in some frequency intervals. The above mentioned properties are usually observable in double negative (DNG) and/or single negative (SNG) media. These kinds of medias can be implemented using split ring resonators (SRRs) ,complementary split ring resonators (CSRRs), thin wires, spiral ,triangular shaped rings and etc.

Studying of anisotropic metamaterials is of great signifycance because of two main reasons: firstly, they exhibit lefthanded behavior in some specific directions of propagation and secondly, their fabrication process is much easier comparing to isotropic metamaterial [9].Here a periodically loaded wave-guide with lossless anisotropic MNG metamaterial is theore-tically analyzed. The waveguide is excited by $T E_{10}$ mode. Using Maxwell's equations and Fluquet's theorem, the dispe-rsion equation is derived. The effect of having different nega-tive values for transversal mu on the wave propagation is discussed using the resulted equation. This can be useful for analysis and better understanding of wave propagation in periodic structures made by metamaterials. This analysis can be useful and applicable for determination and control of pass band or stop-band intervals in filter design. It is worth mentioning

E. Mobini and F. Mohajeri. are with School of Electrical Engineering and Computer Science, Shiraz University, Iran that the zero cutoff frequency of this waveguide contrary to fully filled rectangular waveguide with MNG metamaterial, occurs only for specific ranges of negative transversal permeability and some particular thicknesses of MNG layers. However in the other values of negative transversal permeability the nonzero cutoff frequency may lead to miniaturization effect. The results reveal that the cutoff frequency, dispersion curve, stop band and pass band intervals are highly dependent on the transversal negative mu and also the thickness of MNG layer.

\section{ANALYSIS OF PROPAGATION PARAMETERS}

The rectangular waveguide with transversal dimensions of $\mathrm{a} \times \mathrm{a}$ is assumed to be periodically loaded by layers of uniaxial anisotropic MNG metamaterial (Fig.1).The constitutive para-meters for the MNG layer are stated by the following tensors

$$
\begin{gathered}
\overline{\bar{\mu}}=\mu_{0}(z z)+\mu_{t} \mu_{0}(x x+y y)=\mu_{0}\left[\begin{array}{ccc}
\mu_{t} & 0 & 0 \\
0 & \mu_{t} & 0 \\
0 & 0 & 1
\end{array}\right] \\
\overline{\bar{\epsilon}}=\epsilon_{0}(x x+y y+z z)
\end{gathered}
$$

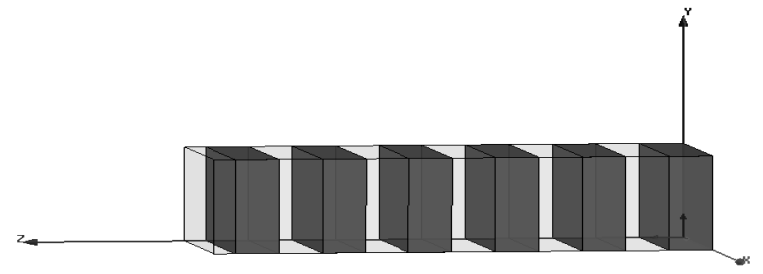

Figure 1. Periodic structure with periodicity of $d_{2}$

Let us assume that electromagnetic fields in the waveguide can be written as:

$E(x, y, z)=E(x, y) e^{-\gamma_{i} z}$

$\mathrm{H}(\mathrm{x}, \mathrm{y}, \mathrm{z})=\mathrm{H}(\mathrm{x}, \mathrm{y}) \mathrm{e}^{-\gamma_{\mathrm{i}} \mathrm{z}}$

where $\gamma_{i}$, the general propagation constant, is equal to $\gamma_{1}$ for air region and $\gamma_{2}$ for $\mathrm{MNG}$ metamaterial region. By solving the vector wave equation in a fully filled waveguide with anisot-ropic metamaterial ( $3 \mathrm{a}$ ), the $\mathrm{z}$ component of magnetic field $(3 \mathrm{~b})$ and also the corresponding dispersion relation can be obtained ( $3 \mathrm{c})$ [7]

$$
\begin{aligned}
& \nabla \times\left({\overline{\overline{\varepsilon_{\mathrm{r}}}}}^{-1} \cdot \nabla \times \mathrm{H}\right)-\omega^{2} \overline{\bar{\mu}} \mathrm{H}=0 \\
& \mathrm{H}_{\mathrm{z}}=\mathrm{H}_{0} \cos \left(\frac{\mathrm{m} \pi \mathrm{x}}{\mathrm{a}}\right) \cos \left(\frac{\mathrm{n} \pi \mathrm{y}}{\mathrm{a}}\right) \mathrm{e}^{-\gamma_{2} \mathrm{z}} \\
& \mu_{\mathrm{z}}=\frac{\mu_{\mathrm{t}}}{\mathrm{k}_{0}^{2} \mu_{\mathrm{t}}+\gamma_{2}^{2}}\left(\frac{\pi}{\mathrm{a}}\right)^{2}
\end{aligned}
$$

By exciting the periodic waveguide and after applying the continuity of tangential electric and magnetic field components at the interface of $M N G$ and air media $\left(z=d_{1}\right)$, 
the following equations are obtained

$$
\begin{aligned}
& E_{y M N G}=\left(A_{1} e^{-\gamma_{2} d_{1}}+A_{2} e^{\gamma_{2} d_{1}}\right) \sin \left(\frac{\pi x}{a}\right) \\
& E_{y \text { air }}=\left(A_{3} e^{-\gamma_{1} d_{1}}+A_{4} e^{\gamma_{1} d_{1}}\right) \sin \left(\frac{\pi x}{a}\right) \\
& \frac{\partial E_{y} M N G}{j \omega \mu_{t} \mu_{0} \partial z}=j \frac{\gamma_{1}}{\omega \mu_{t} \mu_{0}}\left(A_{1} e^{-\gamma_{2} d_{1}}-A_{2} e^{\gamma_{2} d_{1}}\right) \sin \left(\frac{\pi x}{a}\right) \\
& \frac{\partial E_{y \text { air }}}{j \omega \mu_{0} \partial z}=j \frac{\gamma_{2}}{\omega \mu_{0}}\left(A_{3} e^{-\gamma_{1} d_{1}}-A_{4} e^{\gamma_{1} d_{1}}\right) \sin \left(\frac{\pi x}{a}\right)
\end{aligned}
$$

By applying Fluquet's theorem on the transversal fields components at $\mathrm{z}=\mathrm{d}_{2}$, results in the following relations :

$$
\begin{aligned}
& A_{1} e^{-\gamma_{f} d_{2}}+A_{2} e^{-\gamma_{f} d_{2}}=A_{3} e^{-\gamma_{1} d_{2}}+A_{4} e^{\gamma_{1} d_{2}} \\
& \frac{-\gamma_{2} A_{1} e^{-\gamma_{f} d_{2}}}{\mu_{t}}+\frac{\gamma_{2} A_{2} e^{-\gamma_{f} d_{2}}}{\mu_{t}}=-\gamma_{1} A_{3} e^{-\gamma_{1} d_{2}}+\gamma_{1} A_{4} e^{\gamma_{1} d_{2}}
\end{aligned}
$$

where,$\gamma_{\mathrm{f}}$ is the propagation constant for the periodic structure. To have a unique solution for the equations (4)-(5) the determinant of the coefficients matrix should vanish.

$\mathrm{e}^{-2 \gamma_{\mathrm{f}} \mathrm{d}_{2}}+\mathrm{e}^{-\gamma_{\mathrm{f}} \mathrm{d}_{2}}\left[\frac{-1}{\gamma_{1} \gamma_{2}}\left(\frac{\gamma_{2}^{2}}{\mu_{\mathrm{t}}}+\gamma_{1}^{2} \mu_{\mathrm{t}}\right) \sinh \left(\gamma_{1}\left(\mathrm{~d}_{2}-\right.\right.\right.$

$\left.\left.d_{1}\right)\right) \sinh \left(\gamma_{2} d_{1}\right)-$

$\left.\frac{2 \mathrm{j}}{\gamma_{1} \gamma_{2}} \cosh \left(\gamma_{1}\left(\mathrm{~d}_{2}-\mathrm{d}_{1}\right)\right) \cosh \left(\gamma_{2} \mathrm{~d}_{1}\right)\right]+1=$ 0

By considering the expression in bracket as $\mathrm{D}(\mathrm{f})$ the above determinant can be written as(7) $e^{-2 \gamma_{f} d_{2}}+D(f) e^{-\gamma_{f} d_{2}}+1=0$

According to this equation $\mathrm{D}(\mathrm{f})$ is obtained as (8) $D(f)=-2 \cosh \left(\gamma_{\mathrm{f}} \mathrm{d}_{2}\right)$

This solution is valid in both MNG and air media not only in the propagating conditions (when $\gamma_{\mathrm{i}}$ is imaginary) but also for the evanescent case (when $\gamma_{i}$ is real).

Further, if $|\mathrm{D}|<2, \gamma_{\mathrm{f}}$ becomes imaginary which implies a propagating mode for the periodic structure and if $|D|>2$, $\gamma_{\mathrm{f}}$ becomes real and it leads to an evanescent mode.

\section{SIMULATION RESULTS AND DISCUSSION}

In this section parametric study of the structure is presented. This shows that the geometrical parameter and also the transversal mu can highly affect the propagation charac-teristics of the considered periodic structure. The obtained res-ults are then compared to the results of a hollow waveguide.

\section{A. Effect of layer's thickness $\left(d_{1}\right)$}

Figure. 2 shows dispersion curve and $D(f)$ diagram of hol-low waveguide $\left(\mu_{t}=1\right)$ in the frequency region of 0 $100 \mathrm{GHz}$. The transversal dimension of the waveguide is $15 \times 15(\mathrm{~mm})$.

The propagation constant and $\mathrm{D}(\mathrm{f})$ diagram of the periodic structure are depicted In Fig.3. It is obvious that stop band frequency range for $\mathrm{d}_{1}=5(\mathrm{~mm})$ is smaller than $\mathrm{d}_{1}=8(\mathrm{~mm})$. Therefore the cutoff frequency can be controlled by thickness of MNG layer ( the cutoff frequency for $\mathrm{d}_{1}=5 \mathrm{~mm}$ and $\mathrm{d}_{1}=8 \mathrm{~mm}$ are $2.1 \mathrm{GHz}$ and $4.86 \mathrm{GHz}$ respectively) .

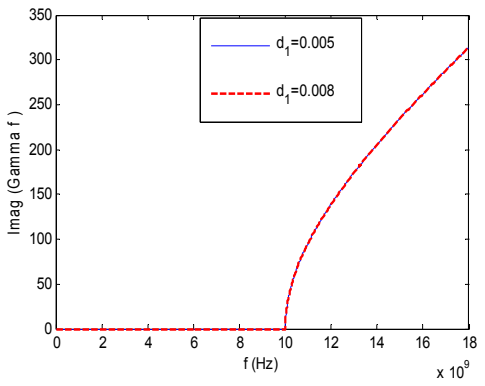

(a)

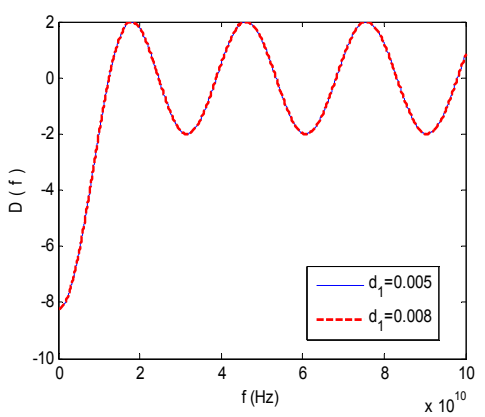

(b)

Figure 2. A hollow waveguide (i.e $\mathrm{Mu}_{\mathrm{t}}=1$ ), (a) $\mathrm{D}(\mathrm{f})$ (b) Dispersion diagram

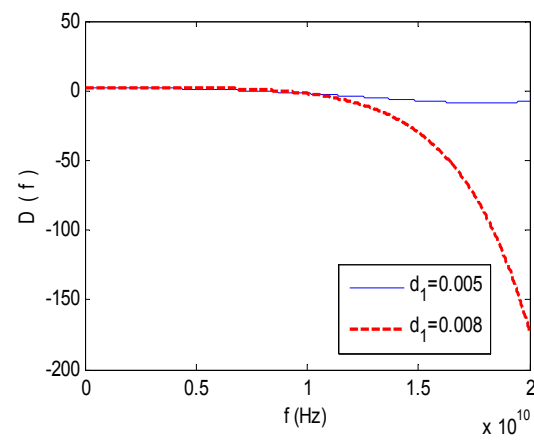

(a)

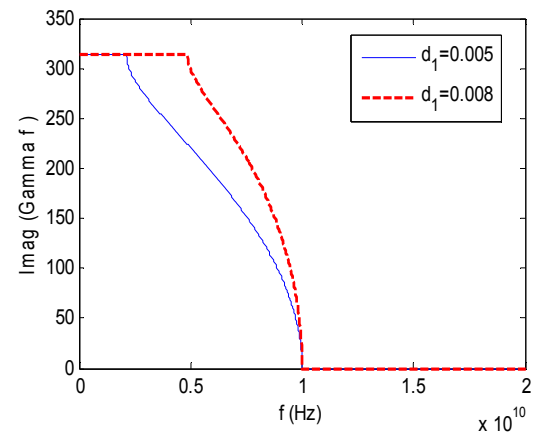

(b)

Figure 3. Combined structure for $\mathrm{Mu}_{\mathrm{t}}=-3$, (a) $\mathrm{D}$ (f) (b) Dispersion diagram

Also the pass band frequency range for $d_{1}=5(\mathrm{~mm})$ is wider than $d_{1}=8(\mathrm{~mm})$. The propagation constant and $D(f)$ diagram of the periodic structure for $\mu_{t}=-0.5$ are depicted In Fig.4. For further investigation, all above simulations are done for transversal permeability $\mu_{\mathrm{t}}=-0.5$ and $\mu_{\mathrm{t}}=-3$ (Fig.5). As it is shown in Fig.5 there is an obvious difference between the obtained results. For example the structure with $\mu_{\mathrm{t}}=-0.5$ has zero cutoff frequency for $\mathrm{d}_{1}=8(\mathrm{~mm})$ but cutoff frequency $\mathrm{f}_{\mathrm{c}}=10 \mathrm{GHz}$ for $\mathrm{d}_{1}=5(\mathrm{~mm})($ Fig. $5-\mathrm{a})$ 


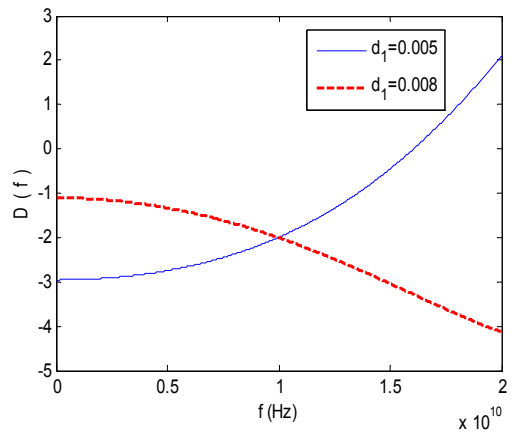

(a)

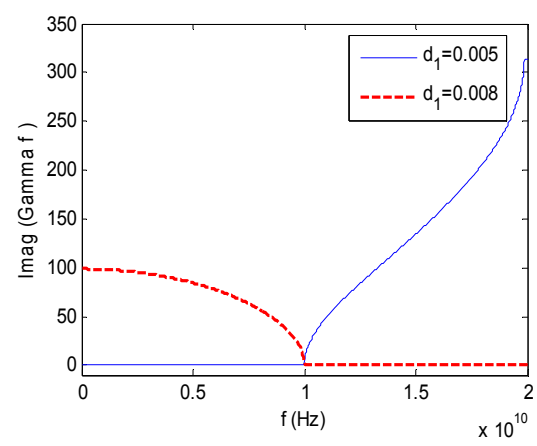

(b)

Figure 4. Combined structure (a) $\mathrm{D}$ (f) (b) Dispersion diagram in 0-20 GHz for $\mathrm{Mu}_{\mathrm{t}}=-0.5$

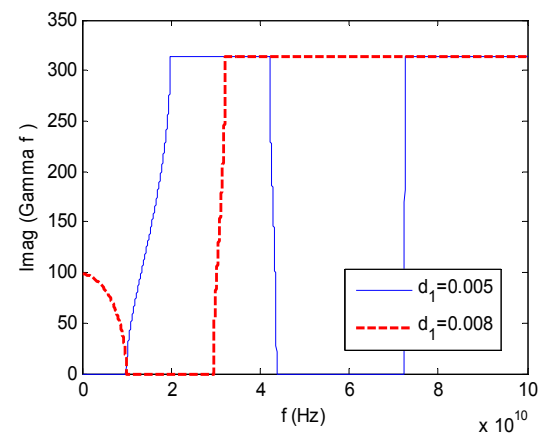

(a)

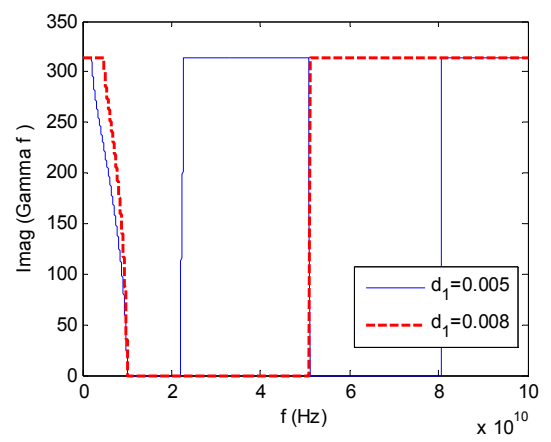

(b)

Figure 5. Combined structure (a) Dispersion diagram in $0-100 \mathrm{GHz}$ for $\mathrm{Mu}_{\mathrm{t}}=-0.5$ (b) Dispersion diagram in $0-100 \mathrm{GHz}$ for $\mathrm{Mu}_{\mathrm{t}}=-3$

\section{B. Effect of transversal mu $\left(\mu_{t}\right)$}

This parameter has strong effect on the cutoff frequency for different layer's thicknesses. Fig.6, exhibits dependency of cutoff frequency on transversal permeability .

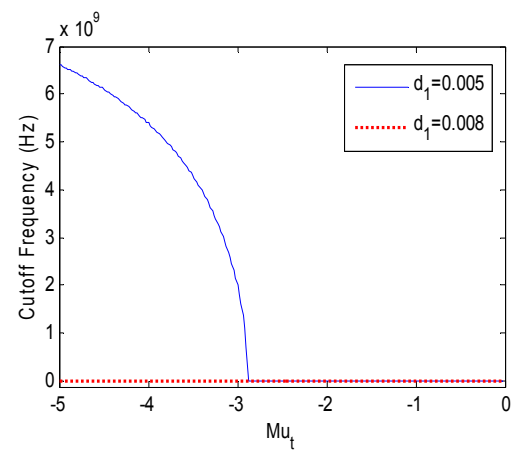

Figure 6. Cutoff frequency of periodic structure versus the value of transversal mufor two different thicknesses of the layers

In the case of $d_{1}=5(\mathrm{~mm})$, the cutoff frequency has slow variation versus transversal negative permeability for $M u_{t} \leq-2.88$. However it has zero cutoff frequency for higher values of $M u_{t}$. For $d_{1}=8(\mathrm{~mm})$, the cutoff frequency is always zero. Therefore, propagation characteristics are depen-dent on the transversal permeability only for some specific layer's thicknesses.

\section{CONCLUSION}

The analysis of the rectangular waveguide loaded with ani-sotropic MNG metamaterial is investigated in this paper. The different behavior in comparison to a hollow structure is discussed. Furthermore; dispersion relation and cutoff frequency curve versus geometrical and magnetic parameter are simulated. Based on these characteristics, the considered structure depending on anisotropic $\mathrm{MNG}$ material and layer's thickness can exhibit various cutoff frequencies, stopband and passband regions.

\section{REFERENCES}

[1] R.Marqués, J.Martel, F.Mesa and F.Medina, "Left-handed media simulation and transmission of EM waves in sub.wavelength SRRloaded waveguides" Phys. Rev. Lett. (2002).

[2] J.D.Baena, R.Marqués, J.Martel, and F.Medina, "Experimental results on metamaterial simulation using SRR-loaded waveguides." IEEE International Antennas and Propagation Symp, Columbus $(\mathrm{OH})$, June 2003.

[3] Silvia Harbar, Juraj Bartolic, Zvonimir Sipus, "Waveguide Miniaturization Using Uniaxial Negative Permeability Metamaterial", IEEE TRANSACTIONS ON ANTENNAS AND PROPAGATION, VOL. 53, NO. 1, JANUARY 2005

[4] S.Hrabar and J. Bartolic, "Backward-wave waveguide based on uniaxial anisotropic negative permeability meta-material," Proc. ICECOM 2003, 2003.

[5] J. Esteban, C. Camacho-Peñalosa, J. E. Page, T. M. Martin-Guerrero, andE. Marquez-Segura "Simulation of negative permittivity and negative permeability bymeans of evanescent waveguide modestheory and experiment." IEEE Trans.Microwave Theory Tech., vol. 53, pp. 1506-1514, 2005

[6] S.N.Burokur,M.Latrach and S.Toutain,Analysis and design of waveguides loaded with split-ring resonators J. of Electromagn. Waves and Appl., Vol. 19, No. 10, 1407-1421, 2005

[7] Fan-Yi Meng, Qun Wu and Le-Wei Li "Transmission characteristics of wave modes in a rectangular waveguide filled with anisotropic metamaterial."Applied Physics A: Materials Science \& Processing ,Volume 94, Number 4 / March, 2009

[8] W. Xu, L.-W. Li, H.-Y. Yao, and T.-S. Yeo Q. Wu "Left-hande material effects on waves modes and resonant frequencies: filled waveguide structures and substrate-loaded patch antennas" J. of Electromagn. Waves and Appl., Vol. 19, No. 15, 2033-2047, 2005

[9] D. R. Smith and D. Schurig "Electromagnetic wave propagation in media with indefinite permittivity and permeability tensors." Phys. Rev. Lett., vol. 90, paper 077405, 2003 
Ehsan Mobini was born in Abadan,Iran, in 1980.He received his BS . degree in telecommunication engineering in 2004 and M.S degree in 2010 from School of Electrical Engineering and Computer Science,Shiraz Unversity,Iran.His research interests include periodic structures, novel $\mathrm{RF} /$ microwave structure and devices, metamaterials.

Farzad Mohajeri was born in 1967 in shiraz,Iran .He received his BS degree in telecommunication engineering in 1991 and M.S degree in 1994 from School of Electrical Engineering and Computer Science,Shiraz Unversity,Iran,and Ph.D degree in 2001 from Tarbiat Modares University,Tehran,Iran .His research interests include antennas and electromagnetic wave propagation in special medias (metamaterials ,chiral and plasma) 